\title{
Deterministic Inverse Design of Lithography-Free, Wavelength-Selective Tamm Plasmon Thermal Emitters
}

\section{Mingze He}

Vanderbilt University https://orcid.org/0000-0001-8773-1268

\section{J. Nolen}

Vanderbilt University

\section{Josh Nordlander}

The Pennsylvania State University

\section{Angela Cleri}

The Pennsylvania State University

\section{Nathan Mcllwaine}

The Pennsylvania State University

\section{Thomas Folland}

The University of lowa

\section{Yucheng Tang}

Vanderbilt University

\section{Bennett Landman}

Vanderbilt University

Jon Maria

Pennsylvania State University

Joshua Caldwell ( $\nabla$ josh.caldwell@vanderbilt.edu )

Vanderbilt University, Mechanical Engineering Department https://orcid.org/0000-0003-0374-2168

\section{Article}

Keywords: Wavelength-selective thermal emitters (WS-EMs), Tamm plasmon polariton (TPP) structures, distributed Bragg reflectors (DBRs)

Posted Date: May 12th, 2021

DOl: https://doi.org/10.21203/rs.3.rs-437911/v1

License: (c) (1) This work is licensed under a Creative Commons Attribution 4.0 International License. 
Version of Record: A version of this preprint was published at Nature Materials on October 21st, 2021. See the published version at https://doi.org/10.1038/s41563-021-01094-0. 


\title{
Deterministic Inverse Design of Lithography-Free, Wavelength-Selective Tamm Plasmon Thermal Emitters
}

Mingze $\mathrm{He}^{1 \dagger}$, J. Ryan Nolen ${ }^{2 \dagger}$, Josh Nordlander ${ }^{3}$, Angela Cleri ${ }^{3}$, Nathan S. Mcllwaine ${ }^{3}$, Thomas G. Folland ${ }^{1,}$ ${ }^{4}$, Yucheng Tang ${ }^{5}$, Bennett A. Landman ${ }^{5}$, Jon-Paul Maria ${ }^{3}$, Joshua D. Caldwell ${ }^{1,5^{*}}$

1. Department of Mechanical Engineering, Vanderbilt University, Nashville, Tennessee, USA

2. Interdisciplinary Materials Science Program, Vanderbilt University, Nashville, Tennessee, USA

3. Department of Materials Science and Engineering, The Pennsylvania State University, University Park, Pennsylvania, USA

4. Department of Physics and Astronomy, The University of Iowa, Iowa City, Iowa, USA

5. Department of Electrical Engineering and Computer Science, Vanderbilt University, Nashville, Tennessee, USA

$\uparrow$ denotes equal contributions

*Email: josh.caldwell@vanderbilt.edu

\begin{abstract}
Wavelength-selective thermal emitters (WS-EMs) are of high interest due to the lack of cost-effective, narrow-band light sources in the mid- to long-wave infrared. Cost-effective WS-EMs can be realized via Tamm plasmon polariton (TPP) structures supported by distributed Bragg reflectors (DBRs) on metal layers, however, optimizing TPP-WS-EMs is challenging because of the large number of parameters to optimize. To address this challenge, we use stochastic gradient descent (SGD) to optimize TPP-WS-EMs composed of an aperiodic DBR deposited on doped cadmium oxide (CdO) plasmonic films. While the SGD-optimized, aperiodic DBR offers extensive spectral control, the material choice, i.e., plasma-frequency-tunable doped $\mathrm{CdO}$, enables the design capabilities not accessible with noble metals. Here, the individual layer thickness and carrier density of $\mathrm{CdO}$ are optimized by our SGD inverse design strategy. The resultant experimental designs demonstrate TPP-WS-EMs exhibiting isolated, high-Q (narrow bandwidth), and structures featuring multiple emission bands for applications such as free-space communications and gas sensing. Furthermore, we illustrate the deterministic design capability within the infrared, such as user-designated Q-factors $(28$ - 10,127) at a desired frequency, multi-band emitters with user-defined Q, and the ability to directly match arbitrary chemical absorption spectra. Thus, the combination of our SGD inverse design and the broadly tunable plasma frequency of $\mathrm{CdO}$ enables lithography-free, CMOS-compatible, and wafer-scale solutions for WS-EMs with unprecedented spectral control.
\end{abstract}




\section{Introduction}

Wavelength-selective thermal emitters (WS-EMs) are of significant interest due to the lack of cost-effective, narrow-band light sources in the mid-infrared, with many applications hindered by the cost and challenging designs of state-of-the-art devices. The majority of proposed WS-EMs are patterned nanostructures ${ }^{1-9}$, typically requiring high-cost, low-throughput lithographic methods. One alternative solution is Tamm plasmon polariton (TPP) heterostructures ${ }^{10}$. Such TPPs consist of a distributed Bragg reflector (DBR) on a conductor, typically a noble metal, where the DBR provides phase-matching to the metal surface, leading to an absorptive resonance with high quality (Q)-factors at normal incident angle ${ }^{10-14}$. As only thin-film deposition is needed to fabricate these structures, TPP-WS-EMs offer a promising platform that can be grown at wafer-scale with relatively low cost, while providing predictable results ${ }^{12-14}$.

Despite the advantages offered by TPP-WS-EMs, applications are hampered due to challenging designs and limitations imposed by the large magnitude of the negative permittivity of the metal employed. To this point, WS-EMs design methodologies have relied on parameter sweeps and physical intuitions. Such design protocols can be applied to periodic DBRs on noble metals, of which the parameter space is limited, and high-Q TPP-WS-EMs can therefore be realized in simple designs ${ }^{13,15}$. However, aperiodic DBRs provide additional spectral control for TPP-WS-EMs, offering the possibility to suppress spurious emission peaks ${ }^{16}$, and achieve ultra-high Q-factors ${ }^{13}$ that are demanding within periodic DBR counterparts. While the aperiodic-DBR TPP-WS-EMs open up more opportunities, this does come at the cost of more challenging designs due to the higher dimensional parameter space. For instance, a parameter sweep for a nine-layeraperiodic DBR infers at least nine-powers-of-ten simulations, which would cost 2,778 hours of processing time assuming 100 simulations per second, and this time grows exponentially with additional layers. Thus, inverse design protocols offer significant advantages, with high-Q TPP-WS-EMs demonstrated at desired wavelengths via Bayesian optimizations ${ }^{12,17}$ and genetic algorithms ${ }^{16}$. However, the optimization efficiency is poor, requiring 24-day simulations on a cluster to optimize one structure ${ }^{12}$, while also restricting design flexibility. Additionally, structures targeting multiple frequencies suitable for multi-gas sensing have not been reported. Furthermore, in experimental reports, only noble metals have been used in TPP-WS-EMs, with plasma frequencies in the visible range, thereby severely restricting the spectral control and making the fabrication not CMOS-compatible.

Here we address all of these challenges, as we develop an inverse design algorithm to efficiently optimize TPP-WS-EMs composed of an aperiodic DBR on an n-doped cadmium oxide (CdO) film. The inverse design protocol is based on stochastic gradient descent (SGD) that allows for the individual layer thicknesses of the DBR, as well as the carrier density (thus the dielectric function) of CdO to be optimized efficiently (minutes on a consumer-grade desktop) with deterministic design capabilities. Experimentally, 
we realize several such inversely designed TPP-WS-EMs targeting narrow-band thermal emission at singleor dual-frequencies for various applications, including filterless nondispersive infrared sensing $\left(\mathrm{NDIR}^{3,18,19}\right.$ ) and high-Q emitters in the long- and mid-wave infrared (LWIR, MWIR), with all structures exhibiting excellent agreement between experiments and simulations. Furthermore, we illustrate precise spectral control of CdO-based TPP-WS-EMs by matching frequencies, lineshapes and amplitudes of arbitrarily shaped spectra across the entire spectral range from the LWIR to telecommunication bands (1550 $\mathrm{nm}$ ), including the ability to individually define the frequency and Q-factors $(27$ - 10,127). In addition, we demonstrate that this approach is capable of matching multiple resonant peaks, for instance, to match absorption spectra of more complex chemicals. Finally, we stress that such functionality is not possible within noble-metal-based TPPs, but instead is enabled by the broadly tunable plasma frequency offered by $\mathrm{CdO}^{20-23}$, which in turn dramatically expands the applicability of WS-EMs. The combination of our efficient inverse-design algorithm and material advancements facilitates the realization of cost-effective, wafer-scale, CMOS-compatible, and lithography-free TPP-WS-EMs for numerous applications, including NDIR, environmental, atmospheric and chemical sensing, free-space communications and IR beacons.

\section{Inverse design protocol}

Our TPP-WS-EMs are comprised of aperiodic DBRs (here, Ge and $\mathrm{AlO}_{\mathrm{x}}$ alternating layers) on thin ( 500 $\mathrm{nm}$ ) $\mathrm{CdO}$ films grown on sapphire substrates. The individual layer thicknesses and carrier density (thus the dielectric function ${ }^{20}$ ) of $\mathrm{CdO}$ are designable parameters, written as a vector $(\vec{t})$. This work employs an SGD-based inverse design technique to determine $\vec{t}$, so that the difference between the absorption spectrum of designed structure (DS) and the target spectrum (TS) is minimized. The design process is initiated by assigning the preferred maximum number of layers for the DBR, with $\vec{t}$ being randomly initialized. Through the transfer matrix method (TMM), DS of the corresponding structure $\vec{t}$ is calculated and compared to TS, therefore resulting in a scalar error. The error is written as a combination of meansquared error (MSE, the first term) and mean absolute error (MAE, the second term):

$$
\text { error }=\operatorname{mean}\left\{\operatorname{ratio} 1 \cdot(\overrightarrow{D S}-\overrightarrow{T S})^{2}+\operatorname{ratio} 2 \cdot|(\overrightarrow{D S}-\overrightarrow{T S})|\right\}
$$

where ratio1 and ratio2 are hyperparameters that can be customized for different purposes (SI, section 9). Note this combination of error is commonly employed in the deep-learning field, and related techniques are powerful for TPP-WS-EM design, such as weighted sampling approaches (SI, section 9). This error is then back-propagated to find the gradient over $\vec{t}$ via SGD. Upon each iteration, the gradient is used to update $\vec{t}$ : 


$$
\overrightarrow{t_{n}}=\overrightarrow{t_{n-1}}-\operatorname{step} \frac{\text { error }_{n-1}}{\partial \overrightarrow{t_{n-1}}}
$$

After each successive iteration, the error is decreased, and the iterations will continue until a predefined maximum number. As such, the structure of the TPP-WS-EM will be optimized to a point where the error between the TS and DS is minimized.

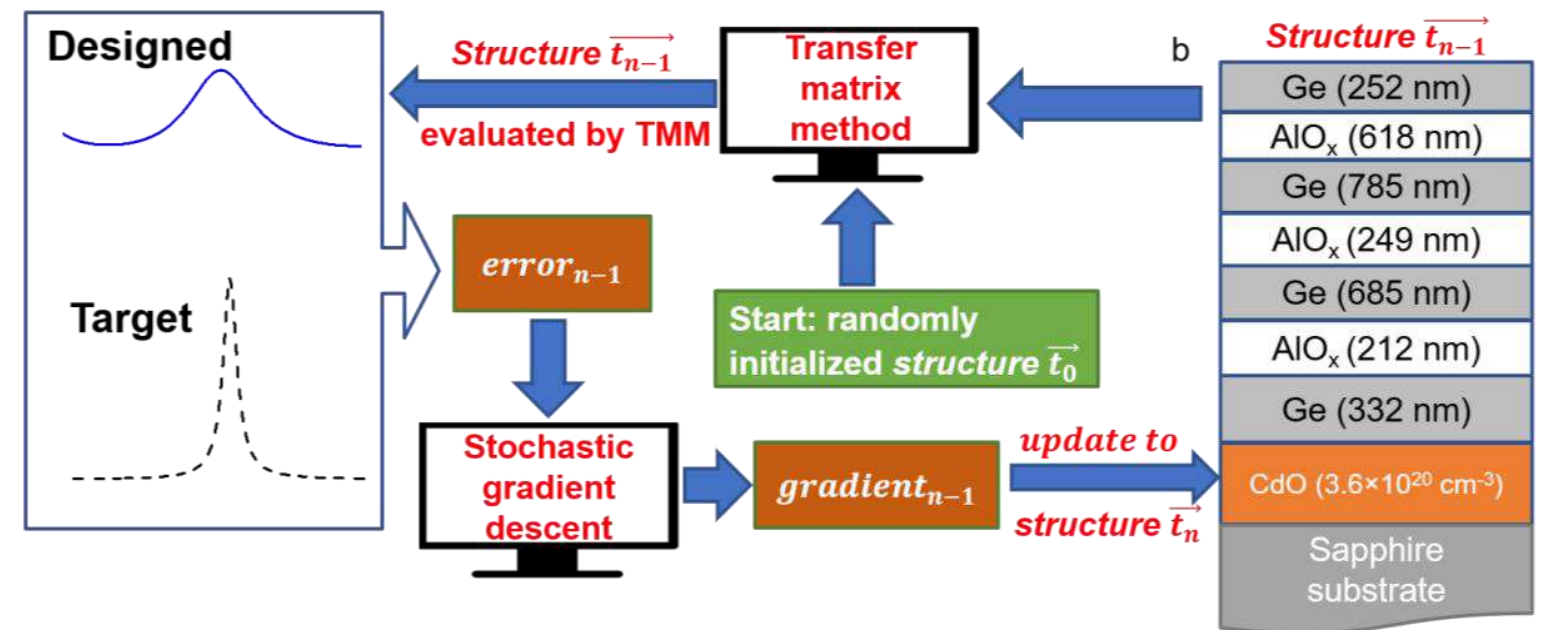

a

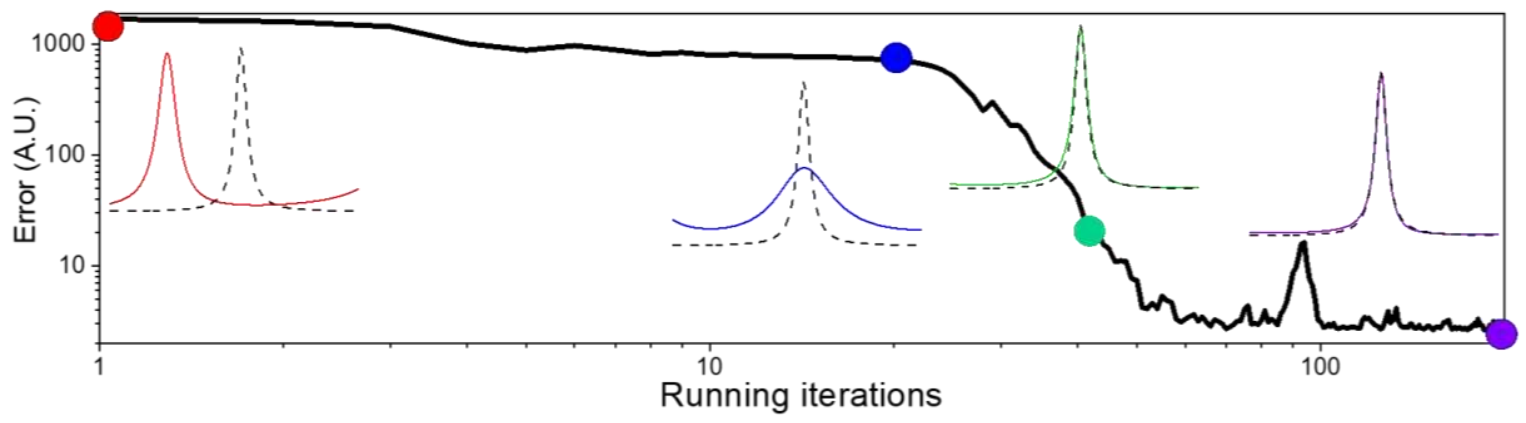

Fig. 1. Flowchart of the design process. The designable parameters of the TPP-WS-EM structure (thickness and carrier concentration), $\vec{t}$, are randomly initialized (green box), then it is evaluated by transfer matrix method (TMM), resulting in the designed spectrum (DS). DS is compared with the target spectrum (TS), leading to a scalar error. In the current iteration (n-1), the gradient of scalar error over $\overrightarrow{t_{n-1}}$, i.e., $\frac{\partial e r r o r_{n-1}}{\partial \bar{t}_{n-1}}$, is calculated by SGD and used to update $\overrightarrow{t_{n-1}}$ to $\overrightarrow{t_{n}}$ by Eq. (2). The updated $\overrightarrow{t_{n}}$ will then be evaluated by TMM, getting a new DS, comparing with TS, updating to a new version, and the process will repeat until a given number of iterations are reached. One exemplary optimizing process is shown in (a), and the optimized structure is shown in panel (b).

While such optimization processes can take many forms, one example is presented in Fig. 1a. Initially, the absorption spectrum of the randomly initialized structure (red solid line) differs significantly from TS. After several iterations, the DS converges towards the TS as the structure is optimized (solid red $\rightarrow$ blue $\rightarrow$ green $\rightarrow$ purple lines in Fig. 1a). Unlike canonical gradient descent calculations used in commercial 
software and previous publications ${ }^{24,25}$, the SGD approach employed here reduces the possibility of getting trapped at poor local minimums and improves the optimization performance ${ }^{26-29}$ (SI, section 4). The exemplary code used in this work is published on our group website ${ }^{30}$.

\section{Experimental demonstration of inversely designed TPP-WS-EMs}

Based on the inverse design algorithm, several TPP-WS-EM structures were designed and fabricated that exhibited a wide range of unique spectral features. The $\mathrm{CdO}$ carrier densities and the designed and as-grown layer thicknesses of these TPP-WS-EMs are provided in SI, section 2, and the growth process is discussed in the Methods. In order to demonstrate the power of inversely designed TPP-WS-EMs, we first provide an experimental device featuring a single, high-Q emission peak. For this effort, we set the TS within one of the atmospheric windows ${ }^{31}\left(2000-3300 \mathrm{~cm}^{-1}\right)$ appropriate for MWIR applications. We model the TS as a flat line with a single, sharp absorption peak centered at $2650 \mathrm{~cm}^{-1}$ and employ the SGD approach to match the DS to the narrowest possible TS (Fig. 2a). Following this optimization, the spectrum of the designed structure exhibits a single peak centered at $2650 \mathrm{~cm}^{-1}$ with a Q-factor of 145 . The experimentally measured emission from the realized structure exhibits a similarly high Q-factor $(Q=128)$, albeit with a resonance that is spectrally shifted to $2721 \mathrm{~cm}^{-1}$. This difference between the DS and the measured spectrum is due to discrepancies between the designed and as-grown DBR layer thicknesses. However, the experimental spectrum agrees exceptionally well with subsequent TMM calculations based on actual, asgrown structure thickness parameters (Fig. 2a. red dashed line). Thus, the spectral offset is the result of the inability to exactly realize the design targets within our deposition chamber, something which would be easily overcome within commercial systems. The experimental Q-factor is on par with the best-reported values using lower loss surface phonon polariton resonators $(\sim 100-400)^{7,32-35}$ and plasmonic Fano resonances $(\sim 100-400)^{36}$. However, we would like to stress that this value is not a fundamental limitation of the TPP-WS-EM design process, but is instead simply a result of the number of DBR layers we used (7 total layers), as well as the material loss of the evaporated $\mathrm{AlO}_{\mathrm{x}}$ (SI, section 1). Later in this work, it is demonstrated that Q-factors over 10,000 are in principle achievable by exchanging $\mathrm{AlO}_{\mathrm{x}}$ for materials with lower losses (such as $\mathrm{ZnSe}$ ) and/or increasing the number of dielectric layers, thereby becoming competitive with 2D photonic crystals, but in a simple, planar multilayer structure.

Besides the previously mentioned transmission window spanning from $2000-3300 \mathrm{~cm}^{-1}$, there is another located within the LWIR $\left(800-1250 \mathrm{~cm}^{-1}\right)$. To demonstrate the broad spectral tunability of CdO-based TMM-WS-EMs, we also inversely designed a TPP-WS-EM with the emission line centered at $1250 \mathrm{~cm}^{-1}$ using the same strategy mentioned above $(\mathrm{Q}$-factor $=16)$. The target, experimentally measured spectrum, and calculations based on designed and as-grown thickness show extraordinary agreements, with all four resonance lines almost overlapped (Fig. 2b). Note that the discrepancy in the spectra is minimal despite 
some differences between the as-grown and designed thicknesses, indicating that some robustness against fabrication error is present.
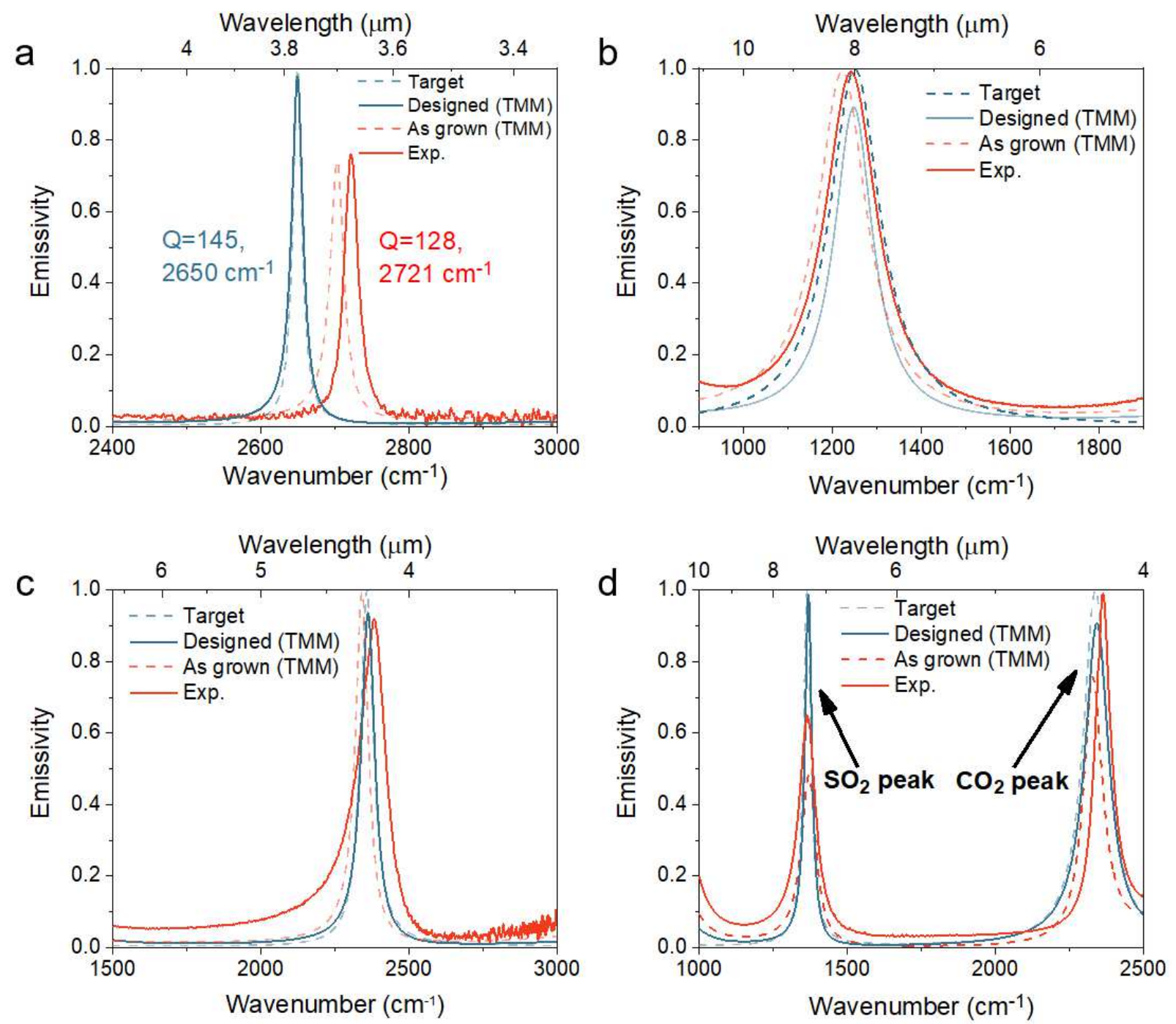

Fig. 2. Experimental demonstration of TPP-WS-EMs. The inverse-design algorithm was employed to realize TMMEM structures featuring (a) High Q-factor emission in the MWIR, (b) single emission mode in the LWIR, and resonant emission designed for NDIR sensing of c) $\mathrm{CO}_{2}$ and d) $\mathrm{CO}_{2}$ and $\mathrm{SO}_{2}$ dual-gas sensing. The target and designed spectra are plotted as blue dashed and solid lines respectively. The emissivity experimentally measured at $150{ }^{\circ} \mathrm{C}$ is plotted as red solid lines, and red dashed lines are calculated absorption spectra of as-grown structures. A photo of the 2inch wafer-scale sample is shown in Fig. S12.

In addition to emitters for line-of-sight applications in these atmospheric windows, the MWIR also contains a large number of unique molecular absorption features, making it of interest for chemical sensing. Thus, 
one application of WS-EMs is filterless NDIR gas sensing ${ }^{3,18,19}$ composed of (1) a WS-EM, (2) a gas cell, and (3) a broadband detector, as shown in SI, section 6. The emission frequency of the WS-EM is centered at the absorption frequency of the gas of interest with a sufficiently high Q-factor to eliminate false-positives resulting from the absorption of other present gases, such as water vapor. To demonstrate the potential of our inversely designed TPP-WS-EMs for NDIR applications, we designed a heterostructure that supports a single emissivity peak that spectrally overlaps with one of the anti-symmetric stretching modes of $\mathrm{CO}_{2}$ $\left(2349 \mathrm{~cm}^{-1}\right)$, while restricting dielectric layer number to three to reduce fabrication complexity. Again, excellent agreement between the TS, DS and experimental structures for the designed TPP-WS-EM is observed, with the measured (target) emission peak centered at $2360 \mathrm{~cm}^{-1}\left(2350 \mathrm{~cm}^{-1}\right)$ with a Q-factor of 21 (40), as shown in Fig. 2c.

So far, we have demonstrated TPP-WS-EM designs with an isolated, tunable peak in emissivity, making these devices suitable for single gas detection in filterless NDIR setups. However, for sensing of multiple gases simultaneously, WS-EMs must possess multiple emission peaks centered at the vibrational absorption resonances of each of those gases, so that different gas concentrations can be evaluated using one device at different temperatures (see derivations in SI, section 11). Yet due to the significant complexity, to the best of our knowledge, WS-EMs with independent design control for each of the distinct emission peaks have not been demonstrated previously. Benefitting from the flexibility of our inverse design protocol, we are able to use an envelope spectrum, which covers both the vibrational modes of $\mathrm{SO}_{2}$ and $\mathrm{CO}_{2}$ as the TS to inversely design a TPP-WS-EM, with the rationale for the TS choice provided in SI, section 10. Here, five dielectric layers were used in order to improve the achievable Q-factors, yielding a DS with two absorption peaks centered at 1367 and $2339 \mathrm{~cm}^{-1}$ (Fig. 2d) that closely matched the amplitudes, peak positions, and full-width-half-maximums (FWHMs) of the TS. The experimental data also agrees well with some minor exceptions, while the center frequencies are closely matched $\left(1358\right.$ and $\left.2360 \mathrm{~cm}^{-1}\right)$, some degradation in the Q-factors and emissivity amplitudes are observed. Nonetheless, this represents the first WS-EM working at designated, disparate frequencies simultaneously. More importantly, with this single TPP-WS$\mathrm{EM}$, the individual concentrations of $\mathrm{CO}_{2}$ and $\mathrm{SO}_{2}$ can be determined simultaneously by linear regression, with more discussions in SI, section 10 that will be the topic of future efforts.

\section{Exploring the potential of inversely designed TPP-WS-EMs}

Despite the limited precision of individual layer thicknesses during film deposition and the additional material losses associated with $\mathrm{AlO}_{x}$, our experimental results reveal the power and potential of inversely designed TPP-WS-EMs, making them suitable for various applications. For advanced WS-EM applications, such as spectral barcoding and multi-gas sensing, user-defined FWHMs and amplitudes at single or multiple frequencies must be realized. Yet, to the best of our knowledge, no designs have been proposed to 
accomplish these tasks. To address these challenges and demonstrate further the potential of the inversely designed TPP-WS-EMs, we explore the design capabilities by utilizing a Ge/ZnSe DBR with 29 layers on a bilayer of $\mathrm{CdO}$ (all designed structures are included in SI, section 8). The bottom CdO layer is constrained to possess the highest achievable carrier concentration to ensure no transmission.

First, we demonstrate the outstanding control over the linewidth at a fixed frequency, presenting structures with Q-factors ranging from 26 to 10,127 with near-unity emission (Fig. 3a). Thus, we demonstrate not only the potential to achieve record-high Q-factors for Tamm-based structures (fitting in SI, Fig. S11), but also the extraordinary ability to match required Q-factors at a given frequency. Such a capability is imperative as different applications have varied requirements for signal-noise-ratio or for matching the absorption spectra to a given molecular vibrational mode. Building on this, we expand upon the potential for such multi-peak designs, providing a TS that exhibits three emission peaks with varying linewidths (Qfactors=25, 37 and 145). Such TPP-WS-EM designs are suitable for spectral barcoding yet have not been demonstrated or proposed, presumably due to the significant design challenges in matching such arbitrary spectral. Despite this difficulty, the DS can be matched to the TS exceptionally well, with only minor discrepancies (Fig. 3b).

Empowered by the widely tunable plasma frequency of $\mathrm{CdO}$ (between $\sim 1200 \mathrm{~cm}^{-1}$ to $7800 \mathrm{~cm}^{-1}$ ), TPP-WSEMs with absorption peaks spanning from short-wave infrared (SWIR) to the LWIR can also be realized. To demonstrate such capabilities, we optimized another TPP-WS-EM structure featuring triple emission peaks, locating at the LWIR $\left(1200 \mathrm{~cm}^{-1}\right)$, MWIR $\left(2700 \mathrm{~cm}^{-1}\right)$ and SWIR $(1.55 \mu \mathrm{m})$ simultaneously (Fig. 3c). Again, the resultant DS matches the TS exceptionally well. We do note that additional modes are present in the spectral regions between these desired emission resonances (SI, Fig. S9), which is a fundamental restriction of the multi-modal nature of the DBR. However, the influence of these additional peaks can be mitigated by the weighted sampling technique (SI, section 9). This demonstration serves to clearly illustrate the unprecedented spectral control afforded by the tunable carrier density of $\mathrm{CdO}$ and the inverse design algorithm employed.

Finally, we emphasize the potential of TPP-WS-EMs in more advanced NDIR applications. Here, it is desirable to match the emitted power of TPP-WS-EMs to the chemical absorption spectra for two reasons: (1) the emitted power should be maximized to suppress the influence of inevitable detector dark-noise, i.e., the TPP-WS-EM should emit energy at all chemical absorption bands; and (2) emitted power is only absorbed by the gas of interest to avoid false-positives, meaning FWHM of TS cannot be larger than the gas spectrum. As the emitted power is temperature dependent and the temperature can be dictated by the application, we generalize this concept by matching the TPP-WS-EM to the chemical absorption spectra. An example is provided for nitric oxide NDIR gas sensing in Fig. 3d. This molecule features multiple 
absorption bands with varying FWHMs and amplitudes between $800-2400 \mathrm{~cm}^{-1}$, making forward-design approaches unrealistic. Yet, our inverse-design algorithm quickly provides an optimized TPP-WS-EM structure with a DS closely matched to the positions, amplitudes and FWHMs of those TS peaks, with undesirable additional modes greatly suppressed (Fig. 3d). The same approach is applied to NDIR greenhouse gas sensings, such as $\mathrm{CO}, \mathrm{O}_{3}, \mathrm{NH}_{3}$, and $\mathrm{CH}_{4}$ shown in SI, section 3. Notably, the shape of the black body emission can be de-convoluted in the design process, so that the emitted power, rather than the emissivity of a TPP-WS-EM can be matched to an arbitrarily shaped target. For an exemplary working temperature $\left(250{ }^{\circ} \mathrm{C}\right)$, we present such an inversely designed TPP-WS-EM of which the emitted power matches the absorption spectrum of $\mathrm{N}_{2} \mathrm{O}$, shown in SI, section 7. We would like to stress again that none of the designs in Fig. 3 have been previously proposed, as the user-defined control of the FWHMs and amplitudes at single/multiple frequencies is not realistic within traditional forward-design approaches. Thus, the design capability highlighted here facilitates WS-EM-based applications for free-space communications, spectral barcoding, multi-chemical sensing with high signal-noise-ratio for highly selective NDIR or alternative gas-sensing metrologies. 

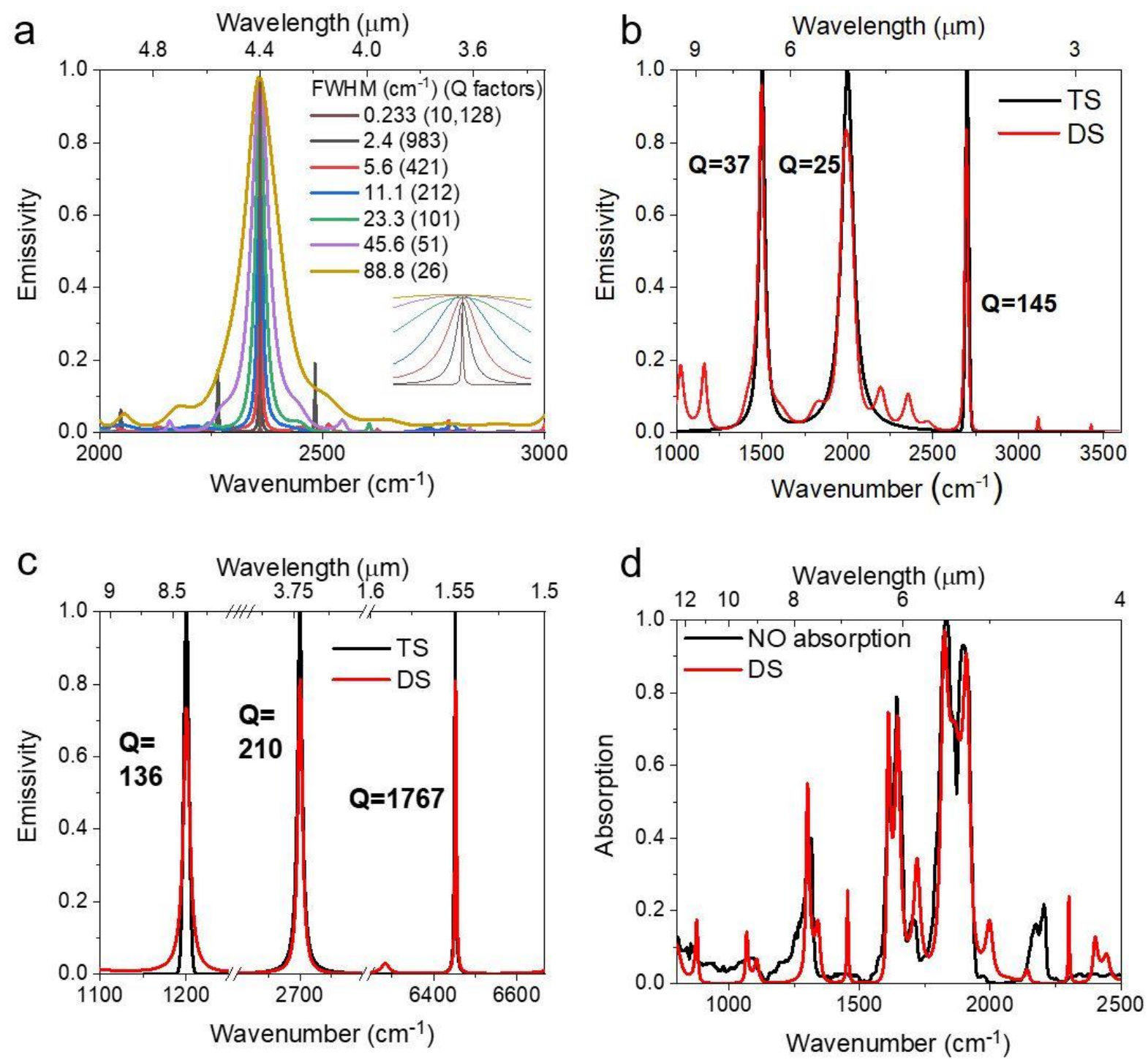

Fig. 3. Inversely designed TPP-WS-EMs for various applications. (a) Isolated emission with different Q-factors. The inset shows near-unity emission for all of them. (b) multiple-peak TPP-WS-EM. (c) One TPP-WS-EM featuring absorption at LWIR, MWIR and telecommunication simultaneously. (d) Matching the absorption spectrum of NO gas for filterless NDIR. The absorption spectrum of NO is normalized to be between 0 and 1 .

\section{CdO: The enabling component for our TPP-WS-EMs}

So far, we have demonstrated the exceptional design freedom offered by our inverse design approach for dictating the emissivity and/or spectrally emitted power of TPP-WS-EM devices. The deterministic design capabilities benefit from two things: the SGD-optimized aperiodic DBRs and the use of doped CdO with a designable dielectric function. The aperiodic DBR exploits the control over the photonic band structure, with the parameter space being limited only by the number of DBR layers. Further, for the structures here, 
the designable dielectric function of $\mathrm{CdO}$ improves this spectral control to an unprecedented level. This additional tuning knob allows for more advanced TPP-WS-EM designs to be realized, such as matching to even more complex absorption spectra. To illustrate this point, we used our SGD-based algorithm to design a CdO-based and a gold-based TPP-WS-EM in an effort to match the absorption spectrum of the nerve agent simulant dimethyl methyl phosphonate (DMMP). Detailed optimizations of the two systems for this purpose are discussed in SI, section 5. Here, an exceptional agreement between the TS and CdO-based TPP-WS-EM design is achieved (Fig. 4a, black and red curves, respectively), including spectral positions, lineshapes and even amplitudes. However, for the gold-based TPP-WS-EM, matching the complex vibrational spectra of DMMP is severely restricted with the same number of dielectric DBR layers (Fig. 4a, blue line). Since the only difference between the two systems is the conductive layer employed (CdO vs. gold), we attribute the significantly improved spectral control to the tunability of $\mathrm{CdO}$ dielectric functions by varying the dopant concentration ${ }^{20-23}$. Note that our inverse design process is fully capable of taking full advantage of this tunability, as it optimizes the carrier concentration as a design parameter without the need for any physical intuition.

To unfold the underlying advantages offered by CdO, we fabricated multiple TPP-WS-EMs composed of the same DBR grown simultaneously on multiple CdO layers featuring different plasma frequencies, i.e., $2700 \mathrm{~cm}^{-1}$ (red dashed curve) and $4300 \mathrm{~cm}^{-1}$ (red solid curve) (Fig. 4b). The emissivity of the low-doped $\mathrm{CdO}$ has several notable differences from that of the higher doped heterostructure. (1) The Tamm resonance frequency is lower and exhibits a higher absorption intensity between 1000 and $2000 \mathrm{~cm}^{-1}$; (2) there is a stronger absorption at the reflection dip of the DBR $\left(\sim 2300 \mathrm{~cm}^{-1}\right)$, which we stress is not a Tamm mode; and (3) no Tamm resonances are supported above the plasma frequency $\left(\sim 3500 \mathrm{~cm}^{-1}\right)$. Thus, the carrier concentration tunability enables additional adjustments of both the frequency and amplitude of the Tamm and non-Tamm absorption modes within these structures. Such an intuitional explanation agrees with the differences observed in Fig. 4a, where the gold-supported structure matches several center frequencies, but not amplitudes and FWHMs. As such, enabled by the combination of the wide tunability of CdO plasma frequency and our inverse design protocol, the design capability of TPP-WS-EMs is increased to an unprecedented level compared with traditional TPP structures using noble metals. In addition to the spectral control offered by $\mathrm{CdO}$, replacing the noble metal with n:CdO also makes the fabrication process CMOS compatible, potentially permitting integrated applications. Notably, our approach can also be applied to other doped materials, such as doped III-V semiconductors ${ }^{37}$ and other transparent conducting oxides ${ }^{38}$. 

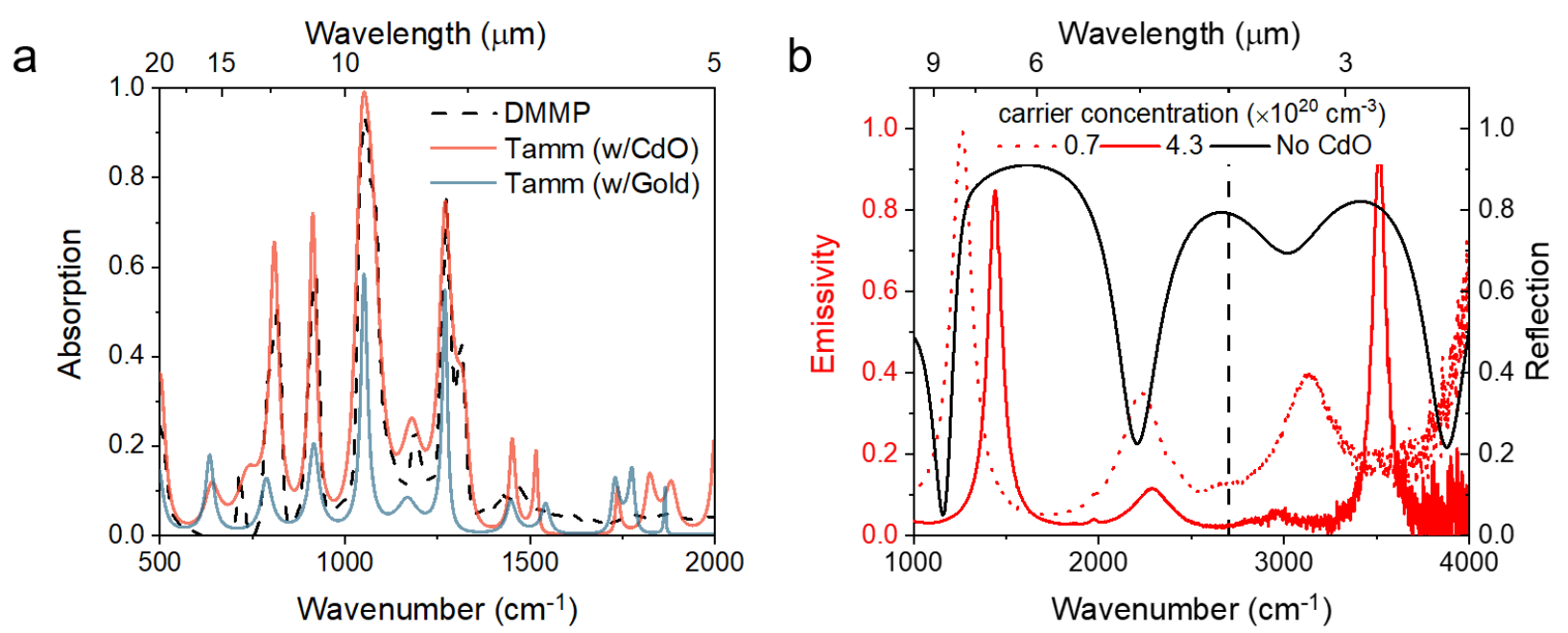

Fig. 4. Functionality enabled by the tunability of CdO plasma frequency. a) TPP-WS-EM designs targeting at DMMP spectrum with $\mathrm{CdO}$ and gold as conductive layers. (b) The emissivity (red lines, experimental data) and reflectance (black lines, calculated by TMM) of the same DBR on CdO of different carrier densities and sapphire substrate. The plasma frequency of $\mathrm{CdO}$ with a carrier density of $7 \times 10^{19} \mathrm{~cm}^{-3}$ is displayed by the vertical dashed line, while the plasma frequency of the other $\mathrm{CdO}$ is above $4000 \mathrm{~cm}^{-1}$.

\section{Conclusion}

In conclusion, the combination of broadly tunable plasma frequency of $\mathrm{CdO}$ and our efficient SGD-based inverse design enables the deterministic design of TPP-WS-EMs, which is numerically and experimentally validated. Using SGD, the structure of TPP-WS-EMs can be efficiently designed (minutes on a consumergrade desktop) with a large degree of design freedom. Equipped with the optimization method, we experimentally demonstrate single- and dual-band TPP-WS-EMs for different applications, including freespace communications, IR beacons and single- and multi-gas filterless NDIR sensing, all showing great agreement between experiments and simulations. Further, we illustrate the unprecedented ability of matching the target spectrum, i.e., the frequencies, FWHMs, and even amplitudes (emissivity or spectral irradiance), by exemplifying several designs ranging from the LWIR to telecommunication band (1.55 $\mu \mathrm{m})$, including isolated emission with user-defined Q-factors (from 28 to 10,127) at a desired frequency, multipeak emission for spectral barcodes and NDIR for matching complex gas absorption spectra. Finally, we stress that such broad functionality is not inherent to TPP-WS-EMs; instead, it is enabled by the exceptionally wide tunability of $\mathrm{CdO}$ plasma frequency. Empowered by our SGD algorithm and this tunability, the demonstrated spectral control of TPP-WS-EMs promises cost-effective, wafer-scale, CMOScompatible and lithography-free solutions for numerous applications throughout the infrared.

\section{Methods}


Device fabrication. In-doped $\mathrm{CdO}$ (n-type) was deposited on 2-inch $r$-plane (012) sapphire single crystal substates at $400^{\circ} \mathrm{C}$ by a reactive co-sputtering process employing high-power impulse magnetron sputtering (HiPIMS) and radio frequency (RF) sputtering from 2-inch diameter metal cadmium and indium targets, respectively. HiPIMS drive conditions were $800-\mathrm{Hz}$ frequency and $80-\mu$ s pulse time, yielding a $1250-\mu$ s period and $6.4 \%$ duty cycle. Film growth occurs in a mixed argon $(20 \mathrm{sccm})$ and oxygen $(14.4 \mathrm{sccm})$ environment at a total pressure of 10 mTorr. Post-deposition, samples were annealed in a static oxygen atmosphere at $635^{\circ} \mathrm{C}$ for 30 minutes.

Dielectric stacks ( $\mathrm{Ge}$ and $\mathrm{AlOx}$ ) were deposited at ambient temperature using electron beam evaporation from Ge (99.999\%) and sapphire sources in vacuum. Thickness was monitored throughout the deposition using a quartz crystal microbalance (QCM). Post deposition, samples were cleaved and the layer thicknesses were measured using cross-sectional SEM.

Thermal Emission Measurements. All the thermal emission were measured at normal incident angle. Thermal emission was measured using a Bruker Vertex 70v FTIR by placing our device on a vertically oriented temperature controller located at the backport of the FTIR. The emission from the sample was then guided and collected through a $\mathrm{KBr}$ window and into the FTIR internal beam path. In this configuration the emitted signal passes through the interferometer block, taking the place of the spectrometer's internal broadband source which is turned off. An aperture was placed in the sample compartment to limit the detected solid angle from the device and reduce the detected emission from within the FTIR. The signal was measured using an IR labs mercury-cadmium-telluride (MCT) detector. In order to calculate the emissivity, thermal emission measurements were collected from our device at $150^{\circ} \mathrm{C}$. These measurements were then compared to the thermal emission measured from an emissivity standard at the same temperature and angle of emission. We used $500 \mu \mathrm{m}$ tall vertically aligned carbon nanotubes (VACNTs) grown on a $\mathrm{Si}$ substrate from an Fe-nanoparticle catalyst, grown by Nanotechlabs Inc., as an emissivity standard ( $\epsilon \sim 0.97)$. These VACNTs provide a high, consistent value for emissivity that is spectrally flat throughout the IR and stable with temperature. The signal collected by the MCT detector in these measurements contains the emission from both the sample as well as the internal optics of the FTIR.

$$
M\left(T_{\text {sample }}, T_{\text {ambient }}, \lambda, \theta\right)=R\left(T_{\text {ambient }}, \lambda\right)\left[S\left(T_{\text {sample }}, \lambda, \theta\right)+G\left(T_{\text {ambient }}, \lambda\right)\right]
$$

Here $M$ is the total measured signal, $R$ is a response function for the internal and external optics, $S$ is the signal originating from the sample and $G$ is the 'background' emission from the internal optics. Thus, in order to isolate the signal from the sample, a background measurement was taken by replacing the sample with a gold mirror. The resultant spectrum is a product of the response function $R$ with the background 
emission $G$. Once the sample, emissivity standard and background emission have been measured we can rearrange equation (3).

$$
\begin{array}{r}
R\left(T_{\text {ambient }}, \lambda\right) S_{\text {sample }}\left(T_{\text {sample }}, \lambda, \theta\right)=M_{\text {sample }}\left(T_{\text {sample }}, T_{\text {ambient }}, \lambda, \theta\right)- \\
R\left(T_{\text {ambient }}, \lambda\right) G\left(T_{\text {ambient }}, \lambda\right) \\
R\left(T_{\text {ambient }}, \lambda\right) S_{\text {standard }}\left(T_{\text {sample }}, \lambda, \theta\right)=M_{\text {standard }}\left(T_{\text {sample }}, T_{\text {ambient }}, \lambda, \theta\right)- \\
R\left(T_{\text {ambient }}, \lambda\right) G\left(T_{\text {ambient }}, \lambda\right)
\end{array}
$$

The response function can be normalized out by taking the ratio of equation (4) to (5) and the emissivity relative to the standard can be determined.

$$
\frac{R\left(T_{\text {ambient }}, \lambda\right) S_{\text {sample }}\left(T_{\text {sample }}, \lambda, \theta\right)}{R\left(T_{\text {ambient }}, \lambda\right) S_{\text {standard }}\left(T_{\text {sample }}, \lambda, \theta\right)}=\frac{S_{\text {sample }}\left(T_{\text {sample }}, \lambda, \theta\right)}{S_{\text {standard }}\left(T_{\text {sample }}, \lambda, \theta\right)}=\varepsilon\left(T_{\text {sample }}, \lambda, \theta\right)
$$

Note that this technique is much quicker than the conventional approach to measuring emissivity using FTIR and allows for multiple background measurements to be taken throughout the day. Therefore, fluctuations in the ambient temperature can be accounted for readily.

Numerical calculation of TPP-WS-EMs. The TMM calculation employed in the inverse design is from textbooks. As the materials are absorbing and dispersive, TMM calculations performed in this paper are from Ref. ${ }^{39,40}$ for cross-validation purposes, and codes are offered by Dr. Nikolai Passler and Prof. Alex Paarmann. The dielectric functions of Ge and AlOx are fitted with ellipsometry measurements with WVase software from J.A. Woolam, $\mathrm{Inc}^{41}$, and temperature-dependent values are adjusted with reflectance data (SI, section 1). The dielectric function model of $\mathrm{CdO}$ with varying carrier concentration is from Ref. ${ }^{20}$ from our groups, and a corresponding MATLAB code to generate $\mathrm{CdO}$ dielectric functions in the MWIR-LWIR is provided on our group website ${ }^{30}$. The outlook section treats the dielectric function of $\mathrm{Ge}^{42}$ and $\mathrm{ZnSe}^{43}$ as constants in the entire frequency range: $16+0 \mathrm{i}$ and $5.0625+0 \mathrm{i}$, respectively.

Absorption data of various chemicals. All chemical absorption spectra are taken from the National Institute of Standards and Technology (NIST) website.

\section{Acknowledgements}

M. H., J. R. N., J-P. M., A. C., and J. D. C. gratefully acknowledge support for this work by Office of Naval Research Grant N00014-18-1-2107. J-P. M. and J. N. acknowledge support from the Army Research Office Research Grant W911NF-16-1-0406. J. N. gratefully acknowledges support from the Department of Defense (DoD) through the National Defense Science and Engineering Graduate (NDSEG) Fellowship 
Program. Y. T. and B. A. L. thank the support from National Science Foundation (NSF 1452485). T.G.F. was supported by Vanderbilt University through J. D. C's startup package. We would like to thank NIST for providing the infrared absorption spectra of chemicals, and Dr. Nikolai Passler and Prof. Alex Paarmann of the Fritz Haber Institute for their TMM code $\mathrm{c}^{39,40}$ to validate our work.

\section{Contributions}

M. H., J. R. N. and J. D. C. conceived the idea. M. H. developed the algorithm. J. R. N. and M. H. performed the infrared measurements. J. N. and A. C. fabricated the samples, and N. S. M. characterized the sample topography. M. H. and Y. C. completed the theoretical analysis regarding the algorithm. All participated in the writing.

\section{Reference}

1 Baranov, D. G. et al. Nanophotonic engineering of far-field thermal emitters. Nature materials, 1 (2019).

2 Lu, G. et al. Narrowband Polaritonic Thermal Emitters Driven by Waste Heat. ACS Omega, doi:10.1021/acsomega.0c00600 (2020).

3 Livingood, A. et al. Filterless Nondispersive Infrared Sensing using Narrowband Infrared Emitting Metamaterials. Acs Photonics.

4 Howes, A., Nolen, J. R., Caldwell, J. D. \& Valentine, J. Near-Unity and Narrowband Thermal Emissivity in Balanced Dielectric Metasurfaces. Advanced Optical Materials 8, 1901470 (2020).

5 Kelley, K. P. et al. Multiple Epsilon-Near-Zero Resonances in Multilayered Cadmium Oxide: Designing Metamaterial-Like Optical Properties in Monolithic Materials. Acs Photonics (2019).

6 Schuller, J. A., Taubner, T. \& Brongersma, M. L. Optical antenna thermal emitters. Nature Photonics 3, 658 (2009).

7 Wang, T. et al. Phonon-polaritonic bowtie nanoantennas: controlling infrared thermal radiation at the nanoscale. Acs Photonics 4, 1753-1760 (2017).

8 Arnold, C. et al. Coherent thermal infrared emission by two-dimensional silicon carbide gratings. Physical Review B 86, 035316 (2012).

9 Asano, T. et al. Near-infrared-to-visible highly selective thermal emitters based on an intrinsic semiconductor. Science advances 2, e1600499 (2016).

10 Kaliteevski, M. et al. Tamm plasmon-polaritons: Possible electromagnetic states at the interface of a metal and a dielectric Bragg mirror. Physical Review B 76, 165415 (2007).

11 Sasin, M. E. et al. Tamm plasmon polaritons: Slow and spatially compact light. Applied physics letters 92, 251112 (2008).

12 Sakurai, A. et al. Ultranarrow-band wavelength-selective thermal emission with aperiodic multilayered metamaterials designed by Bayesian optimization. ACS central science 5, 319-326 (2019).

13 Wang, Z. et al. Ultra-narrow and wavelength-tunable thermal emission in a hybrid metal-optical Tamm state structure. Acs Photonics (2020). 
Wang, Z. et al. Narrowband thermal emission realized through the coupling of cavity and Tamm plasmon resonances. Acs Photonics 5, 2446-2452 (2018).

15 Yang, Z.-Y. et al. Narrowband wavelength selective thermal emitters by confined tamm plasmon polaritons. Acs Photonics 4, 2212-2219 (2017).

16 Botros, J., Ali, M. O., Tait, R. N., Amaya, R. E. \& Gupta, S. Direct thermal emission testing of aperiodic dielectric stack for narrowband thermal emission at mid-IR. Journal of Applied Physics 127, 114502 (2020).

17 Zhang, W., Wang, B. \& Zhao, C. Selective Thermophotovoltaic Emitter with Aperiodic Multilayer Structures Designed by Machine Learning. ACS Applied Energy Materials (2021).

18 Lochbaum, A. et al. On-chip narrowband thermal emitter for mid-IR optical gas sensing. Acs Photonics 4, 1371-1380 (2017).

19 Lochbaum, A. et al. Compact mid-infrared gas sensing enabled by an all-metamaterial design. Nano letters 20, 4169-4176 (2020).

20 Nolen, J. R. et al. Ultraviolet to far-infrared dielectric function of $\mathrm{n}$-doped cadmium oxide thin films. Physical Review Materials 4, 025202 (2020).

21 Liu, C. P. et al. Effects of free carriers on the optical properties of doped CdO for full-spectrum photovoltaics. Physical Review Applied 6, 064018 (2016).

22 Runnerstrom, E. L., Kelley, K. P., Sachet, E., Shelton, C. T. \& Maria, J.-P. J. A. P. Epsilon-near-zero modes and surface plasmon resonance in fluorine-doped cadmium oxide thin films. 4, 1885-1892 (2017).

23 Sachet, E. et al. Dysprosium-doped cadmium oxide as a gateway material for mid-infrared plasmonics. Nature materials 14, 414-420 (2015).

24 Xue, W. \& Miller, O. D. High-NA optical edge detection via optimized multilayer films. arXiv preprint arXiv:2101.03160 (2021).

25 Jiang, J. \& Fan, J. A. Multiobjective and categorical global optimization of photonic structures based on ResNet generative neural networks. Nanophotonics 1 (2020).

26 Bordes, A., Bottou, L. \& Gallinari, P. SGD-QN: Careful quasi-Newton stochastic gradient descent. Journal of Machine Learning Research 10, 1737-1754 (2009).

27 Sohl-Dickstein, J., Poole, B. \& Ganguli, S. 604-612 (PMLR).

28 Robbins, H. \& Monro, S. A stochastic approximation method. The annals of mathematical statistics, 400-407 (1951).

29 Kingma, D. P. \& Ba, J. Adam: A method for stochastic optimization. arXiv preprint arXiv:1412.6980 (2014).

30 https://my.vanderbilt.edu/caldwellgroup/.

31 https://atran.arc.nasa.gov/cgi-bin/atran/atran.cgi. Empty Empty, Empty, doi:Empty.

32 Caldwell, J. D. et al. Sub-diffractional volume-confined polaritons in the natural hyperbolic material hexagonal boron nitride. Nature communications 5, 5221 (2014).

33 Caldwell, J. D. et al. Low-loss, extreme subdiffraction photon confinement via silicon carbide localized surface phonon polariton resonators. Nano letters 13, 3690-3697 (2013).

34 Tamagnone, M. et al. High quality factor polariton resonators using van der Waals materials. arXiv preprint arXiv:1905.02177 (2019).

35 Autore, M. et al. Boron nitride nanoresonators for phonon-enhanced molecular vibrational spectroscopy at the strong coupling limit. Light: Science \&Amp; Applications 7, 17172, doi:10.1038/Isa.2017.172

https://www.nature.com/articles/Isa2017172\#supplementary-information (2018).

36 Wang, B. et al. High-Q Plasmonic Resonances: Fundamentals and Applications. Advanced Optical Materials, 2001520 (2021). 
37 Law, S., Adams, D. C., Taylor, A. M. \& Wasserman, D. Mid-infrared designer metals. Optics express 20, 12155-12165 (2012).

38 Bikbaev, R. G., Vetrov, S. Y. \& Timofeev, I. V. Transparent conductive oxides for the epsilon-nearzero Tamm plasmon polaritons. JOSA B 36, 2817-2823 (2019).

39 Passler, N. C. \& Paarmann, A. Generalized $4 \times 4$ matrix formalism for light propagation in anisotropic stratified media: study of surface phonon polaritons in polar dielectric heterostructures. JOSA B 34, 2128-2139 (2017).

40 Passler, N. C., Jeannin, M. \& Paarmann, A. Layer-resolved absorption of light in arbitrarily anisotropic heterostructures. Physical Review B 101, 165425 (2020).

41 https://www.jawoollam.com/ellipsometry-software/wvase.

42 Burnett, J. H., Kaplan, S. G., Stover, E. \& Phenis, A. 99740X (International Society for Optics and Photonics).

43 Gao, W. $72832 \mathrm{~L}$ (International Society for Optics and Photonics). 


\section{Supplementary Files}

This is a list of supplementary files associated with this preprint. Click to download.

- TammSIsubmissionApril172021.docx 